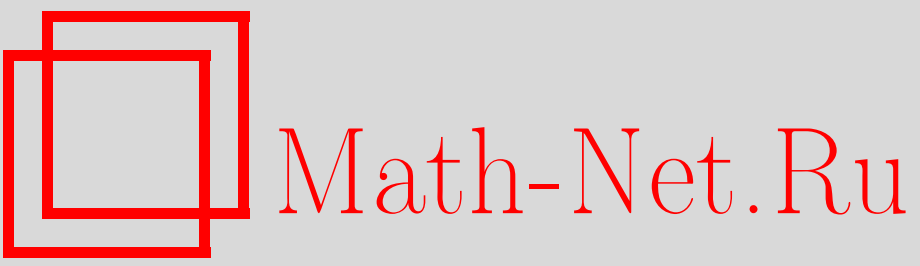

В. А. Пунинская, Гипотеза Воота для модулей над наследственными нетеровыми первичными кольцами, УМH, 1999, том 54, выпуск 6, 171-172

DOI: https://doi.org/10.4213/rm243

Использование Общероссийского математического портала Math-Net.Ru подразумевает, что вы прочитали и согласны с пользовательским соглашением

http://www . mathnet.ru/rus/agreement

Параметры загрузки:

IP: 54.197 .130 .99

26 апреля 2023 г., 13:48:14 


\title{
ГИПОТЕЗА ВООТА ДЛЯ МОДУЛЕЙ НАД НАСЛЕДСТВЕННЫМИ НЕТЕРОВЫМИ ПЕРВИЧНЫМИ КОЛЬЦАМИ
}

\author{
В. А. ПуНИНСКАЯ
}

Эта статья является продолжением работы автора [1]. Полное доказательство результатов, сформулированных в ней, будет опубликовано в [2]. Гипотеза Воота утверждает, что для любой счетной (полной) теории $T$ первого порядка число неизоморфиных счетных моделей $T$ либо счетно, либо континуум. Гипотеза Воота для $\omega$-стабильных теорий модулей была доказана Каравалья [3; теорема 6]. Полным $n$-типом в теории $T(n<\omega)$ называется совместное с $T$ полное множество формул от $n$ свободных переменных без параметров. Множество всех $n$-типов теории $T$ обозначается $S_{n}(T)$. Все кольца, рассматриваемые в этой статье, предполагаются счетными. Модуль (над счетным кольцом $R$ ) называется модулем с малым числом типов, если для любого натурального $n$ его (полная) теория $\operatorname{Th}(M)$ имеет только счетное число $n$-типов.

Пусть $M$ не является модулем с малым числом типов. Так как для любой счетной полной теории $T$ либо $\left|S_{n}(T)\right|=\omega$, либо $\left|S_{n}(T)\right|=2^{\omega}$ [4; теорема 6.3.4], то $\operatorname{Th}(M)$ имеет континуум $n$-типов. Поскольку каждая счетная модель теории $\operatorname{Th}(M)$ не может реализовать более чем счетное число $n$-типов, $\operatorname{Th}(M)$ имеет $2^{\omega}$ неизоморфных счетных моделей и гипотеза Воота верна для $\operatorname{Th}(M)$. Следовательно, в силу результата Каравалья гипотеза Воота выполняется для произвольной полной теории модулей над любым кольцом, над которым каждый модуль с малым числом типов является $\omega$-стабильным.

ПрЕДЛОЖЕнИЕ 1. Пусть $R$ - счетное простое полунаследственное справа нётерово справа кольцо, имеющее конечную левую размерность Голди. Тогда каждый правый $R$-модуль с мальм числом типов $\omega$-стабилен.

Напомним, что кольцо $R$ имеет классическое правое кольцо частных, если множество его регулярных элементов является правым множеством Оре.

ПрЕДЛОЖЕНИЕ 2. Пусть $R$ имеет классическое правое кольцо частных $Q(R)$. Если гипотеза Воота верна для произвольной полной теории модулей над $Q(R)$, то она верна для произвольной полной теории модулей без кручения над $R$.

ДоКАЗАТЕЛЬСТво. Пусть $M$ - правый модуль без кручения с малым числом типов. По [5; следствие 1.3] $M$ делим. Тогда $M$ становится правым модулем над $Q(R)$ при определимом действии последнего.

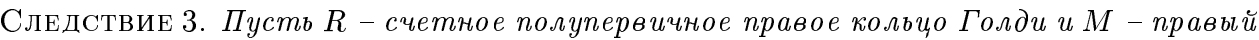
$R$-модуль без кручения с малым числом типов. Тогда $M$ является $\Sigma$-инвективным, следовательно, $\omega$-стабильным модулем.

Основным результатом этой статьи является

Теорема 4. Каждый модуль с малым числом типов над счетным наследственныцм нётеровым первичным кольцом $\omega$-стабилен.

В [6; теорема 3.2] доказывается аналогичная теорема для счетных (некоммутативных) дедекиндовых первичных колец, т.е. таких наследственных нётеровых первичных колец, которые не содержат ненулевых собственных идемпотентных идеалов [7; 5.6.3]. Представлялось интересным убрать в этом результате ограничение на число ненулевых собственных идемпотентных идеалов, что и удалось сделать в предыдущей теореме. Из теоремы 4 немедленно получаем

СледСтвиЕ 5. Гипотеза Воота верна для произвольной полной теории модулей над счетнылм наследственным нётеровым первичным кольцом.

Автор пользуется случаем поблагодарить профессора А. В. Михалева за помощь и интерес к данной работе. 


\section{СПИСОК ЛИТЕРАТУРЫ}

[1] Прест М., Пунинская В.А.// УМН. 1999. Т. 54. № 3. С. 149-150. [2] Puninskaya V. Modules with few types over a hereditary Noetherian prime ring // J. Symbolic Logic (to appear). [3] Garavaglia S. // J. Symbolic Logic. 1980. V. 45. P. 155-164. [4] Hodges W. Model Theory. Cambridge: Cambridge Univ. Press, 1993. [5] Puninskaya V. Vaught's conjecture for modules over a serial ring // J. Symbolic Logic (to appear). [6] Puninskaya V. // Bull. London Math. Soc. 1999. V. 31. № 2. P. 129-135. [7] McConnell J. C., Robson J. C. Noncommutative Noetherian Rings. New York: Wiley, 1987.

Московский государственный

Принято редколлегией университет им. М. В. Ломоносова

02.10.1999 